\title{
Enseignement laïque, porosité religieuse et lutte de légitimité dans l'enceinte scolaire française en Tunisie.
}

\author{
Émilie Pontanier \\ Laboratoire Interdisciplinaire des Solidarités, Sociétés, Territoires (UMR 5193) \\ Centre d'Anthropologie Sociale \\ Université Toulouse le Mirail.
}

\section{Résumé}

Comment la laïcité des lycées français en Tunisie permet, dans les murs de l'enceinte scolaire, de légitimer la vérité religieuse ou areligieuse des lycéens tunisiens et binationaux ? Comment sert-elle la pluralité des pratiques religieuses des familles qui y scolarisent leurs enfants ? Au travers d'une analyse qualitative des choix scolaires motivés par des stratégies d' «entre-soi » social et religieux, l'article montre que ce système d'enseignement favorise, d'une part, l'autonomie cultuelle des familles musulmanes et, d'autre part, le rejet de l'islam dogmatique. La laïcité est alors analysée comme une valeur qui permet le contournement des codes sociaux et religieux du pays. En effet, pour la majeure partie des lycéens et des familles des établissements français, la laïcité autorise une pluralité de pratiques religieuses et, surtout, la préservation de leur vision de l'islam dans une société qui révèle d'autres pratiques et d'autres valeurs et qui engendre des craintes d'embrigadement. 
Comment les lycéens tunisiens et binationaux des établissements français ${ }^{1}$ concilient-ils leurs allégeances à la modernité laïque d'une part et à la modernité religieuse d'autre part ? De quelles manières l'enceinte de l'école leur permet-elle de résister au prosélytisme et à l'imposition dogmatique de quelques camarades et de quelques enseignants ? Comment l'enseignement français contribue-t-il à entretenir leur désir de distinction sociale et cultuelle?

Nous allons montrer que l'espace scolaire est instrumentalisé par des parents d'élèves et des lycéens pour entretenir un «entre-soi » social et religieux et se préserver d'une religion plus rigoriste que la leur. Pour cela, ils se servent du terrain laïque comme d'un champ de forces et de luttes pour faire contrepoids et refuser d'autres visions du monde et d'autres « vérités religieuses ». La préservation des croyances religieuses ou areligieuses dans une société qui tend à radicaliser les devoirs religieux passe ainsi par le biais d'un enseignement laïque.

Apparentés à des espaces de clôture sociale (Van Zanten, 2009a.) et de clôture religieuse, les établissements français permettent de mettre les élèves à l'abri des pratiques religieuses qui tendent à s'imposer dans l'espace public mais aussi dans l'institution publique qui se trouve confrontée à une demande idéologique des jeunes scolarisés pour plus de religiosité (Melliti, 2008). Le chômage, le désœuvrement et l'absence de libertés individuelles sous le régime du président Ben Ali ont entraîné un redéploiement des modes de religiosité des jeunes générations qui a abouti au déclin de l'islam institutionnel (ibid.: 70). Les difficultés sociales ont ainsi laissé se créer une brèche religieuse dans laquelle les groupes radicaux se sont engouffrés et "la religiosité a gagné en intensité ou, pour le moins, en visibilité et en véhémence chez les jeunes maghrébins » (ibid. : 65).

La démocratisation de l'enseignement tunisien a effectivement doté les jeunes d'une autonomie linguistique qui leur a permis d'interpréter le Coran, tout en se libérant de l'autorité institutionnelle ${ }^{2}$. En outre, le groupe de pairs a aussi joué un rôle dans la socialisation islamiste au sein des institutions scolaires et universitaires, ce qui a participé à la radicalisation d'une partie de la population (ibid. : 65).

Dans ce contexte, se diriger vers un lycée français permet de contrer l'influence islamiste puisque l'établissement reste soumis aux principes généraux de l'enseignement laïque même si l'espace scolaire est davantage inclusif qu'exclusif dans son rapport au religieux. En effet, les fêtes nationales et religieuses tunisiennes sont intégrées au calendrier scolaire et un aménagement horaire en période de ramadan est mis en place. Malgré cela, les établissements restent conformes à leurs principes laïques avec, par exemple, le refus des signes ostentatoires, comme le port du «voile», et la condamnation du prosélytisme religieux.

Si la question du port du voile ne se pose pas dans les murs de l'école, l'institution scolaire reste toutefois confrontée au problème du prosélytisme lorsque des lycéens remettent en cause les fondements de la laïcité en faisant preuve de véhémence religieuse auprès des non-croyants, des non-pratiquants ou des «mauvais musulmans ». Parallèlement, certains enseignants ne respectent pas leur devoir de réserve en introduisant des discours partisans dans la relation pédagogique.

Puisque certains élèves ou enseignants remettent en cause la "vérité religieuse » ou areligieuse de la population scolaire, des lycéens et des familles utilisent la dénonciation pour tenter de faire valoir les prescrits laïques sur les prescrits religieux dans un espace scolaire qui

\footnotetext{
${ }^{1}$ L'établissement secondaire français de Tunis compte 33 nationalités qui se répartissent ainsi : 895 élèves tunisiens, 94 français, 588 binationaux franco-tunisiens, 30 autres nationalités représentées par des effectifs de 1 à 13 élèves (AEFE, $2010: 26)$.

${ }^{2}$ Chez les musulmans, les oulémas (savants) sont les docteurs de la loi ou l'autorité religieuse et judiciaire. Ils ont pour fonction d'expliquer le Coran et de présider aux exercices de la religion.
} 
permet de négocier le rejet dogmatique et la réislamisation de la société. La dénonciation consiste à fournir aux personnels de direction (délégués des professeurs, membres de l'administration) des informations sur l'élève ou le professeur prosélyte : le «transgresseur » qui remet en cause la liberté de conscience de chacun.

\section{1- Méthodologie}

Le matériau empirique mobilisé s'appuie sur une étude de terrain située auprès des deux établissements secondaires français de Tunisie. Cette étude a été réalisée avant la Révolution de jasmin de 2011. Le recueil de données compte cent trente-neuf entretiens individuels et collectifs (enseignants, corps encadrant, surveillants, parents d'élèves et lycéens). Dans cet article, des noms d'emprunts ont été utilisés pour chaque passage d'interview cité.

L'enquête s'est construite autour d'une perspective ethnosociologique et chaque hypothèse de travail s'est progressivement consolidée au fur et à mesure des observations, des entretiens formels et informels et des récits de vie (Bertaux 2005 [1997]).

\section{2- Les transgresseurs ou des lycéens aux référents religieux et identitaires}

La majorité des lycéens des établissements français s'inscrivent en faux contre la mutation religieuse et contre le mode de religiosité qu'ont adopté certains de leurs congénères du système public tunisien. Dans leur ensemble, les lycéens et les familles s'opposent à une lecture fondamentaliste de l'islam et à sa visibilité ostentatoire. C'est la raison pour laquelle nous avançons que la laïcité conforte leur distinction sociale et culturelle car, dans les murs de l'école, elle sert de rempart contre le prosélytisme et elle appuie leur désir de modernité religieuse. En effet, comme la laïcité autorise une pluralité de conceptions religieuses, les lycéens peuvent adopter des pratiques «accommodantes », voire une absence de pratiques religieuses, puisqu'ils aspirent à fixer des limites à la religion. Ils se soustraient ainsi aux visées de l'islam prosélyte et se libèrent de ses contraintes dans l'espace public. L'espace éducatif favorise alors l'accomplissement d'un islam «accommodant » et permet aux lycéens de prendre leur indépendance cultuelle. Nous entendons par religiosité accommodante, une individualisation des pratiques religieuses qui renvoie à une lecture religieuse souple, modérée et peu exigeante qui implique l'autonomie du croyant.

\subsection{Islam prosélyte versus autonomie et indépendance religieuses}

Dans ces établissements français, quelques lycéens anciennement scolarisés dans le système public tunisien transgressent toutefois la laïcité. Ces lycéens sont peu nombreux et leur nombre peut varier de 1 à 6 dans une classe ${ }^{3}$ en fonction de l'option internationale du baccalauréat ${ }^{4}$. Ces élèves, parfaitement arabophones, ont intériorisé les codes d'une culture scolaire et religieuse qui diffère de celle de l'enseignement français. Leur acclimatation aux codes de la laïcité et aux codes sociaux adoptés par les lycéens « occidentalisés » est donc parfois problématique, notamment parce que leur rapport à l'islam diverge.

\footnotetext{
${ }^{3}$ En classe de seconde, le nombre d'élèves dans une classe avoisine les 30 lycéens. En première et en terminale ES et S, leur nombre oscille entre 20 et 28 , en section L, entre 10 et 20.

${ }^{4}$ L'option internationale du baccalauréat (OIB) implique une similitude d'enseignement avec le cursus arabisé de l'enseignement public tunisien de la seconde à la terminale. Le statut particulier de l'OIB permet la poursuite des études dans l'enseignement supérieur aussi bien français, étranger que tunisien.
} 
«Par exemple, j'ai un élève ici qui vient du Ministère. Il est habitué depuis tout petit au Coran, alors sur ses copies, il met des prières. Je lui ai expliqué qu'ici, ça ne se faisait pas, mais pour nous c'est naturel, d'accord?» (Monsieur Ben Amar, professeur d'arabe).

Des lycéens nouvellement inscrits se réfèrent souvent à la parole de Dieu puisqu'elle guide leurs comportements sociaux. Ils la mobilisent aussi comme un schème de pensée et une donnée irréfutable parce que «leurs dispositions incorporées génèrent le plus souvent des pratiques symboliques systématiques » (Bourdieu, 1980) rarement remises en cause par un système d'analyses objectif.

«Dans les classes d'OIB, y a toujours besoin de remettre en place cette valeur de laïcité. Il est difficile de leur faire accepter que l'argument religieux n'est pas un argument justement. [...] Et je vais stopper le discours si je sens qu'il y a un peu trop de prosélytisme ou quelque chose comme ça. Mais finalement, je suis plus tolérante que la classe [Rires] qui parfois ne va rien laisser passer: Madame, punissez-le, etc.» (Madame Martin, enseignante de français).

Ce qu'exprime Madame Martin et, avec elle, d'autres enseignants français, c'est la difficulté que rencontrent certains de ces lycéens à s'adapter au mode scolaire français, notamment parce que jusque-là le regard critique et analytique ne faisait pas partie de leur fonctionnement scolaire. Les enseignants se montrent tolérants à l'égard des dérapages religieux de ces lycéens travaillés par les référentiels de l'islam. En revanche, leur attitude incite le reste de la population scolaire à tracer une ligne de démarcation qui permet de préserver un «entre-soi » séculier. Ils sont stigmatisés et exclus, notamment parce que leur rapport à l'islam entraîne des querelles et des conflits de légitimité religieuse.

Les lycéens issus du système public tunisien appartiennent tendanciellement au même milieu économique que la population scolaire des établissements français. En revanche, ils ne partagent pas systématiquement leur biculturalité, ni les mêmes codes socioculturels. Ils sont également désorientés par la religiosité distinctive des lycéens. Par exemple, le manquement au jeûne du mois de ramadan est, pour certains, une des limites morales et religieuses qu'ils ne peuvent concevoir.

Maya, une lycéenne italo-tunisienne de 16 ans, en seconde, s'insurge contre la pression que subissent les non-pratiquants dans la sphère publique et insiste sur la capacité de l'enceinte scolaire à libérer les lycéens du carcan religieux.

«Moi je remarque davantage le coté sournois de certains [dans la rue] parce que je suis pas pratiquante. Comme je suis Tunisienne de moitié, ils me disent : honte sur toi! Et, par exemple, si on mange, on nous regarde d'un air, $j$ 'sais pas quoi, c'est pas agréable! Heureusement, au lycée, on est tranquille, si on te fait une réflexion, tu peux aller te plaindre à la vie solaire. [Une réflexion ?] Oui, si on te critique parce que tu bois ou si tu mets du rouge à lèvre ou autre, c'est interdit. Bon mais les nouveaux le savent pas mais ils apprennent très vite parce que c'est pas agréable d'avoir une sanction [Rires] ! »

À l'abri de la sphère publique, les lycéens peuvent déplorer la porosité religieuse entre public et privé, désapprouver le prosélytisme et condamner la réprobation qui est adressée aux non-pratiquants. Ils peuvent légitimer leurs prises de positions antidogmatiques et contrecarrer 
la pénétration prosélyte. Pour y parvenir, ils dénoncent les fauteurs de trouble à l'administration.

Les non-pratiquants sont, pour la plupart, des lycéens tunisiens ou binationaux qui sont musulmans; seule une minorité se considère comme athée. Les uns et les autres veulent contrôler leur liberté religieuse et leur liberté de ne pas avoir de religion. Et c'est sur le terrain scolaire que ces enjeux vont se nouer. En effet, les établissements français sont le terrain de conflits plus ou moins dissimulés entre les tenants de l'autonomie religieuse et ceux de l'encadrement dogmatique. Ils mettent ainsi à jour la concurrence et le rapport de force continuel entre eux.

Bien que l'enclave laïque entretienne les intérêts spécifiques de la population scolaire en l'affranchissant des codes sociaux et religieux de la sphère publique et en autorisant, un temps, la remise en cause de l'ordre dominant, celui-ci reprend très vite ses droits aux abords des établissements.

«Et des fois quand il y a ramadan, plus maintenant, mais à un moment, y a eu des bagarres devant le lycée parce que ceux qui mangent vont dans la petite cafétéria en face. Bon, ils achètent des sandwiches. Et une fois, ils ont mangé dans la rue. Bon, ça a bien bardé [...]. Le lycée devrait mettre des surveillants dehors quandmême. Il faut faire en sorte que les alentours du lycée soient surveillés par du personnel du lycée et pas par des policiers hein? Préserver simplement la tranquillité de nos enfants, au prix où est la scolarité ! Maintenant, les enfants restent dans la petite cafète pour manger tranquillement, ils n'osent plus sortir. $C$ 'est navrant. » (Madame Zriba, Franco-Tunisienne, mère d'élèves).

Aux abords des établissements, «la rue » reprend ses droits. De fait, les lycéens qui dérogent aux règles religieuses dans l'espace public, notamment en période de ramadan, peuvent être harcelés moralement, voire pourchassés par des dogmatiques. En se réfugiant dans l'enceinte scolaire, ils peuvent alors défendre leurs positions et inverser le rapport de force, ils peuvent également compter sur l'appui des parents d'élèves lorsqu'ils cèdent et battent en retraite devant le dogmatisme.

«Moi j'suis athée donc y a des trucs comme ça que je n'arrive pas à saisir. Je ne comprends pas les gens qui jugent par rapport à la pratique religieuse alors qu'on est tous de la même nationalité, on vit dans le même pays. C'est Tunisien qui compte, pas musulman. [...] Pendant le ramadan, ceux qui ne mangent pas et qui nous voient renter dans le self acheter un sandwich, ils nous disent : c'est pas bien, tu cherches à nous tenter, pourquoi tu manges ça devant moi ? Bon, à force de l'entendre, moi, vraiment des fois, je me dis, j'aurai dîu renter manger chez moi. Quand je dis ça à mes parents, ils ne sont pas d'accord [Rires]! Tu n'as pas à plier me dit mon père : t'es dans un lycée français, tu as droit à tes libertés !» (Sonia, Tunisienne, 18 ans, terminale S).

Sonia s'insurge contre le caractère « hégémonique " de l'islam qui tend à minimiser le rôle de la nationalité et de la culture tunisiennes comme lien social au profit d'une unité fondée uniquement sur la religion. Malgré cela, elle se laisse harceler passivement. Interpelés, ses parents interviennent pour lui rappeler son droit à une liberté de conscience. C'est une manière, pour eux, de contrer l'autoritarisme de l'islam et de s'opposer à son influence.

Tantôt dominante, tantôt dominée, la population scolaire est alors soutenue et secondée par les parents d'élèves pour interpeler les transgresseurs et pour exclure le prosélytisme des murs des établissements, sans pour autant parvenir à endiguer complètement le phénomène. Le contrôle parental contribue ainsi à négocier la sauvegarde de la liberté de conscience des élèves et la sauvegarde de l'espace laïque. 


\subsection{Islam identitaire des Français musulmans et légitimité de la laïcité}

En France, les enfants issus de l'immigration maghrébine ont parfois mobilisé la religion et ses signes ostensibles d'appartenance pour pénétrer l'espace laïque et pour conjurer l'exclusion sociale et les ségrégations sociales et ethno-raciales dont ils sont les victimes. En Tunisie, les établissements français scolarisent une infime minorité de lycéens français d'origine tunisienne dont les parents sont revenus s'installer «au pays ». Ces lycéens n'appartiennent pas au même milieu socioéconomique que celui de la population scolaire et la plupart bénéficie d'une bourse scolaire. En France, ils convoquaient l'islam pour affirmer leur authenticité, leurs racines tunisiennes et pour marquer leur différence avec les Français. En Tunisie aussi, l'autodéfinition de ces élèves, héritée de leur expérience en France, continue de passer par l'accomplissement religieux, la religion ayant pour eux une fonction «identificatoire » (Bordes-Benayoun, Tandian, 2004). Mais se revendiquer de l'islam en Tunisie perd de son sens en raison du caractère national de la religion. De plus, leurs conceptions et leurs pratiques religieuses, considérées comme étant «d'un autre âge » par la population scolaire, les excluent et font qu'ils sont, à nouveau, stigmatisés et méprisés. Ils continuent de subir une forme de violence symbolique au travers du mépris et du racisme social qu'ils suscitent. Ils sont d'abord repérés par leur façon de parler caractéristique des banlieues, ensuite identifiés en fonction de leur origine («beurs des quartiers sensibles »), et parfois discriminés en raison de leur ferveur religieuse jugée « rétrograde ». Pourtant ils s'imaginaient qu'ils pourraient, dans leur pays d'origine, extérioriser et afficher librement leur attachement à l'islam sans souffrir de résistances. Leur retour avait été idéalisé et la réalité à laquelle ils sont confrontés dans les établissements français ne correspond pas à ce qu'ils espéraient : ils subissent, une nouvelle fois, une stigmatisation religieuse couplée à un racisme de classe.

Si certains parviennent à s'adapter à ce nouvel environnement social, d'autres perpétuent des actes d'incivilité qu'ils justifient par la religion et blâment leurs «frères musulmans » pour être aussi peu respectueux de l'islam. Leurs propos prosélytes aboutissent à des conflits qui leur font prendre conscience que l'expression de leur appartenance à l'islam était plus aisée dans le système scolaire public en France.
«Il faut savoir qu'entre Tunisiens de France et Tunisiens de Tunisie, y a une forme de racisme, une sorte de malaise réel. Ils s'insultent, y a une forme de cloisonnement, d'isolement, de persécution entre eux. [...] En plus, ce sont généralement ces élèves là, les Tunisiens qui arrivent de France, qui posent problème scolairement. » (Madame David, professeur d'Anglais).

Alors que l'islam, dans les cités, s'est révélé être un «élément fédérateur » (ibid.: 173), il est, dans ce nouvel espace scolaire, un élément qui divise les musulmans car la majorité a pris de la distance avec les revendications prosélytes et la pratique effective. En effet, la volonté d'autonomisation religieuse de la majorité contrecarre les objectifs des « identitaires » dont les violences verbales ou physiques sont dénoncées au nom de la liberté de conscience. Toutefois, les obstacles à leur intégration ne sont pas réductibles qu'à leur prosélytisme; ils sont également liés à leur milieu socioéconomique. En effet, les rapports sociaux entre la population scolaire et certains de ces «identitaires » sont liés à une distance sociale et culturelle plus ou moins radicale qui permet à la population scolaire de s'opposer aussi à leurs origines communes et à leurs points communs.

Ces élèves issus de l'immigration sont également vus comme une menace par les parents d'élèves qui présentent ces jeunes comme des intrus malvenus. Madame Djil, Tunisienne, une mère d'élèves de 46 ans, femme au foyer et membre de l'association des parents d'élèves, explique : 
«Les Tunisiens qui ont vécu en France et qui viennent ici, eux, leur intégration est difficile et pour eux et pour les autres. [...] Bon, ils ne sont pas très bien élevés. D’ailleurs, ils ont pas mal de problèmes scolaires aussi. Ils sont même paumés dans la religion. [Que voulez-vous dire ?] Ils n'ont pas de repères, ils ont avalé des couleuvres, passez moi l'expression! Y a un décalage avec le lycée. On les reconnaît déjà par rapport au langage... et par rapport à la religion aussi. Heu, ils sont musulmans bien sûr mais c'est un islam plus poussé que le nôtre. D'ailleurs, nous sommes assez vigilants à l'association, vigilants envers leurs écarts de comportements. [Il me semble que ces élèves sont très minoritaires ?] C'est-à-dire que même si c'est un ou deux, il suffit de laisser faire et c'est la brèche, vous comprenez? Et puis, c'est un lycée laïque ici, y a des règles, on n'est pas en ZEP. »

L'existence de différences irréductibles entre la population scolaire et les élèves issus des banlieues françaises défavorisées rend l'apparition de pratiques ségrégatives légitime bien que regrettable (Van Zanten, 2009a.). Ces différences sont liées à leur mauvais niveau scolaire et à leur milieu social d'origine. En outre, en exhibant l'islam comme une identité, ils suscitent une insécurité religieuse qui incite les parents d'élèves à faire preuve de vigilance pour préserver un entre-soi de classe (ibid.). En effet, la domination sociale des familles constitue un moyen d'ostracisme des enfants de milieu populaire. En voulant préserver les frontières laïques des établissements français, les parents cherchent surtout, avec les ressources dont ils disposent, à protéger et à préserver leur mode de vie et leur distinction cultuelle.

La «diabolisation» des autres «différents de soi »a aussi vocation à préserver le modèle de l'«intégration par cloisonnement» (Van Zanten, 2009b.) et en attribuant à ces élèves des rôles sociaux (Payet, 1999), les parents affichent leur méfiance et leur volonté de limiter tout contact avec ces lycéens «identitaires » qui seraient «contaminés » par une autre interprétation de l'islam et cela aboutit effectivement à leur mise à distance de la vie scolaire.

La laïcité est ainsi mobilisée pour rejeter la règle prosélyte et elle est instrumentalisée pour servir les intérêts spécifiques des familles en matière religieuse et sociale, car elle s'avère un précieux allié pour conduire l'islam à retrouver sa vocation religieuse. En effet, la position sociale des familles, les conduites occidentalisées des lycéens et les valeurs portées par la laïcité conduisent "l'islam à retrouver sa nature véritable et sa vitalité proprement religieuse » (Barbier, 1995 : 212).

\section{3- Luttes de légitimité éducative entre les vérités laïques et les vérités religieuses}

Des lycéens sont nombreux également à refuser les opinions religieuses de leurs enseignants et, souvent, les savoirs de ces derniers sont discrédités en raison de leur position dogmatique. En effet, certains professeurs recrutés localement, comme des professeurs d'arabe ou d'éducation physique et sportive se servent de la relation pédagogique pour prêcher les valeurs de l'islam, le plus souvent sans se rendre compte de la transgression commise, compte tenu du caractère englobant de leur vision religieuse. Ainsi, si les professeurs de nationalité française (en contrat résident ou expatrié) associent le plus souvent la laïcité à des valeurs de tolérance et de respect et considèrent que c'est le guide d'une formation critique et objective, à l'inverse, pour des enseignants de nationalité tunisienne (en contrat local), ces valeurs sont précisément celles de l'islam, et la transmission des savoirs ne peut se faire que sous sa gouverne.

Les enseignants prosélytes introduisent donc des prescrits religieux dans la relation pédagogique et ceux-ci servent de principes d'autorité. Ils sont happés par leur habitus 
religieux et la transmission des savoirs souffre dès lors d'un mode d'inculcation inadapté et elle peut engendrer une lutte de légitimité éducative entre les acteurs de l'espace laïque, la population scolaire et les parents d'élèves.

\section{3-1 Libertés religieuses versus dogmatisme}

En effet, l'islam légitime leur orientation pédagogique comme une ressource mise au profit des valeurs à transmettre aux élèves. Ils utilisent les textes du Coran et n'hésitent pas à faire apprendre des sourates dans leurs cours.

«Ici [au lycée français], c'est pas accès sur la religion mais en cours d'arabe on parle quand même de notre religion. On a des textes du Coran, on a des devoirs religieux mais c'est normal les élèves qui font arabe sont musulmans. [...] Si parfois des élèves demandent des explications, par exemple, sur la religion, d'autres élèves disent que le lycée est laïque. Mais y en a qui exagèrent avec la laïcité, ils sont contre mes cours juste parce que j'ai mis des sourates alors ils sont contre l'étude. [Dictaphone coupé] Vous savez j'ai eu des parents très mécontents à cause de ça. Ils m'ont convoqué et je dois faire attention. Mais je ne savais pas qu'il ne fallait pas parce que moi je reprends les cours du Ministère [enseignement public tunisien] [Reprise de l'enregistrement]. Bon, mais maintenant j'ai compris... Il faut savoir que les parents ont toujours un regard sur les cours qu'on fait, toujours. Les parents savent tout. L'année dernière un parent m'a dit qu'il n'aime pas un texte qu'on fait en cours, alors je l'ai remplacé cette année. » (Monsieur Tabarki, professeur d'arabe).

Si la laïcité implique la liberté religieuse, elle suppose également un devoir de réserve pour l'enseignant. Des lycéens ont assimilé ce principe absolu de neutralité dans l'enseignement laïque et ils attendent des enseignants qu'ils s'y conforment. Or celui-ci ne semble pas avoir conscience des limites que suppose la laïcité, puisque ses convictions religieuses s'immiscent dans la relation pédagogique et la dominent. C'est ainsi que la valeur de respect devient un gage de reconnaissance mutuelle, tiré non pas de la laïcité mais de l'islam et du Coran.

Ces enseignants, forts du bien-fondé de leur action pédagogique et de leur posture religieuse, ne parviennent pas toujours à saisir déontologiquement leurs manquements. $\mathrm{Ce}$ sont des lycéens, relayés par leurs parents, qui entreprennent alors la sauvegarde des principes laïques en tentant de faire reculer le prosélytisme religieux. Les enseignants capitulent le plus souvent, généralement en raison de la position sociale des familles et de leur réseau d'influence. En effet, des parents désapprouvent farouchement toute entorse faite à leur autonomie religieuse et c'est avec une grande familiarité qu'ils brandissent la bannière de la laïcité auprès de l'administration pour qu'elle recadre les enseignants prosélytes ou, le cas échéant, qu'elle les renvoie pour incompatibilité de valeurs.

Les pratiques pédagogiques de ces professeurs sont donc dénoncées parce qu'elles sont effectivement répréhensibles dans un cadre laïque, mais elles servent aussi de prétexte aux lycéens pour dominer socialement ces enseignants. En effet, les lycéens instrumentalisent leur connaissance du cadre laïque pour renforcer leur domination sociale sur des enseignants qui ne parviennent pas à intérioriser les codes du système scolaire dans lequel ils évoluent. Par exemple les élèves revendiquent leur liberté de manger en période de ramadan en invoquant la laïcité et les parents, à leur tour, l'invoquent lorsque les propos religieux des enseignants outrepassent leur devoir de réserve. 
«On est Tunisien, on est des arabes d'accord, on n'a pas le droit de parler du christianisme [dans l'enseignement tunisien], ça peut jouer contre toi un jour, les parents peuvent porter plainte en disant que le prof parle de christianisme. [...] Au début j'ai cru que la laïcité c'était ce qui était contre la religion islamique et par la suite j'ai vu que l'on peut parler de la religion islamique et d'autres religions. [...] Voilà, la religion c'est personnel, je te parle dans l'absolu bien sûr, il faut convaincre les gens pour qu'ils te suivent ou pour qu'ils soient convaincus. Ça c'est notre devoir à tous [Rires], le devoir de tout bon musulman!» (Monsieur Ben Amar, professeur d'arabe).

La laïcité est parfois assimilée à un principe qui irait à l'encontre de l'islam. Cette représentation est probablement liée au contexte international et est associée plus particulièrement à la France en raison de sa position contre le foulard à l'école. C'était peutêtre également, à l'époque de l'enquête, une manière de critiquer indirectement la position du gouvernement de Zine el Abidine Ben Ali avec sa campagne anti-hijab (voile que les femmes se mettent sur la tête en laissant le visage apparent) et de désavouer sa conception politicoreligieuse. Cette représentation est également le fruit d'une méconnaissance puisque des enseignants admettent avoir découvert les principes de la laïcité, notamment par le biais de la connaissance objective des religions monothéistes, élément inconcevable dans le système public tunisien.

Les devoirs religieux de ces enseignants priment et leur devoir de réserve est systématiquement entamé. Leur habitus religieux domine la relation pédagogique et se heurte à celui des lycéens. L'espace scolaire laïque se transforme alors en terrain de lutte pour la réalisation légitime de l'islam des uns et des autres. Leïla, une lycéenne tunisienne de terminale ES nous confie :

«Par exemple, quand je leur donne mon avis [aux professeurs d'arabe], ils disent qu'on ne pense pas ce qu'il faut, qu'il faut lire le Coran, tu vois hein? Pas plus tard que l'autre jour, mon prof d'arabe me fait une réflexion de ce genre. C'est pas à lui de dire quoi que ce soit. Ça non! Jamais. Il critique pas ma foi. Moi, franchement, j'ai un Coran, c'est comme mon porte-bonheur mais je vais pas m'engager dans une lecture et tout... Je déteste quand il fait ça. L'année dernière ma prof, elle était pas comme ça. Elle était plus ouverte. »

La transgression des enseignants consiste à empiéter sur la liberté de croyance des lycéens en cherchant à diriger leur foi et en se substituant ainsi à un imam. Malgré le refus affiché de certains lycéens, il est notable que l'islam prosélyte arpente l'espace laïque pour accueillir de nouveaux adeptes. L'islam prosélyte est alors combattu par des lycéens qui se réfèrent à l'islam lui-même et à la laïcité pour faire valoir leur droit à la liberté de conscience.

Si la laïcité permet parfois de tenir à distance l'islam prosélyte, elle est également instrumentalisée pour amoindrir l'autorité professorale des enseignants tunisiens qui ont généralement une attitude moralisatrice plus que dogmatique ; ils sont néanmoins condamnés par les parents d'élèves qui remettent en cause toute l'autorité du maître.

«J'ai choisi une école laïque, il faut qu'elle le reste. [C'est-à-dire ?] C'est simple, vous avez vu les professeurs d'arabe? Moi je le dis à l'administration, il ne faut pas qu'ils mélangent savoir et dogme, c'est compris !? [...] C'est à la cellule familiale de s'occuper de la religion et je ne veux pas qu'il y ait d'interférence entre Dieu et mes enfants. Si j'avais voulu j'aurais pu les mettre à la Zeïtouna qui est une école coranique, d'accord ? Le lycée français doit veiller à sa laïcité. » (Madame Abdallah, Tunisienne, mère d'élèves). 
Madame Abdallah a suivi une scolarité dans le système scolaire français, à Tunis. Elle est professeur d'université et son mari est ingénieur. Ils sont musulmans non-pratiquants et ils contestent l'encadrement religieux de l'enseignement public tunisien qui va de pair avec la réislamisation en cours de la société. Ils ont choisi l'enseignement laïque notamment pour préserver l'autonomie religieuse de leurs deux enfants. C'est pour cela que Madame Abdallah contrôle le contenu des cours de ses enfants et veille au devoir de réserve des enseignants qui seraient tentés de les endoctriner. Choquée par l'étalage des convictions privées de quelques enseignants qui remettent en cause la neutralité de l'enseignement, elle fait valoir les droits de la laïcité auprès de l'administration et court-circuite les ambitions dogmatiques potentielles des enseignants concernés en mobilisant ses différentes formes de capital.

\section{3-2 Résistances et contrepoids des familles}

L'objectif de ces parents est donc de dominer l'islam prosélyte et de promouvoir leur «vérité » et leur autonomie religieuse par le biais de la laïcité. Ils vont jusqu'à retirer leurs enfants du cours d'arabe lorsque le programme étudié va à l'encontre de l'éthique familiale.

«Le professeur d'arabe essaye de donner quelques indications sur la religion musulmane du pays ça me paraît un peu logique, mais au niveau historique. À partir du moment où il commence à donner des consignes de pratiques religieuses, là je suis plus réticente. [Ça arrive?] C'est arrivé à une parente d'élève, une amie à moi. Une Française mariée à un Tunisien qui a refusé que son fils continue d'aller à un cours d'arabe parce que c'était trop porté, à une période de l'année, sur le Coran. Alors elle en a parlé, elle ne trouvait pas ça normal. Nous sommes allées à l'association des parents d'élèves pour être guidées et retirer nos enfants de ce cours d'arabe. Mais les profs d'arabe trouvent ça tellement normal de vanter la parole de Dieu que pff [«soupir»]. $C$ 'est le problème en Tunisie, la religion s'intensifie mais ici nous sommes sur un îlot. Et puis, nous sommes privilégiés ce qui facilite nos prises de position.» (Madame El Jery, Française, mère d'élèves mariée à un Tunisien).

Usant de leur capital économique, culturel, social ou symbolique, certains parents peuvent s'immiscer dans la relation pédagogique et user de leurs atouts pour affaiblir l'islam qu'ils récusent. Ils ont fait le choix de l'enseignement français par conviction idéologique, or, les sommations religieuses auxquelles leurs enfants sont confrontés empêchent la transmission d'un savoir objectif et le développement de l'esprit critique.

Les parents usent ainsi de leur position sociale pour discréditer le capital sur lequel repose la force de leur adversaire, généralement le capital religieux, et valoriser le capital dont ils sont particulièrement pourvus (Bourdieu, Wacquant 1992: 75). Cela incite des parents d'élèves à intervenir auprès de l'administration pour dénoncer les agissements religieux des enseignants et pour participer à leur renvoi. Madame Kadihamine, une sophrologue tunisienne, mariée à un artiste peintre, témoigne :

«J'ai choisi une école française car ça correspond à ma mentalité. Je suis profondément attachée à la laïcité et à la modernité. [...] Moi je suis musulmane et mes fils aussi, ils n'ont pas reçu d'éducation religieuse puisqu'ils sont au lycée français, mais les grands-parents ont pris soin de leur rappeler les fondamentaux. Et, voilà, j'ai eu un problème avec un professeur d'arabe : un extrémiste. J'ai fait ce qu'il fallait avec l'association de parents d'élèves, il a été viré purement et simplement. Il n'est pas question qu'on vienne embrigader nos enfants, je ne suis pas d'accord. Et lui, il trouve que nos enfants ne pensent pas correctement et 
qu'il faut les remettre dans le droit chemin. On rêve! On trouve plus de problèmes avec les professeurs d'arabe, ils n'ont pas la même mentalité, pas les mêmes convictions et ça laisse des traces dans leurs cours, il faut être vigilant.»

Laïque et musulmane, Madame Kadihamine revendique l'application stricte de la neutralité afin de préserver l'objectivité des savoirs transmis et favoriser l'autonomie de l'élève puisque la laïcité ne souhaite «ni prouver, ni démontrer, ni convaincre. C'est comprendre. Comprendre par le dialogue, comprendre pour pouvoir dialoguer » (Bouchet, 1996). Sa lutte contre le prosélytisme aboutit à la remise en cause du mode pédagogique de certains enseignants puisqu'ils ne dissocient pas le mode religieux du mode laïque.

L'apparition de l'islam prosélyte dans les établissements français rappelle aux familles l'hégémonie de l'islam dans la sphère sociale et son difficile contournement. Comme l'espace scolaire permet de s'opposer à cette hégémonie, des parents se servent de la laïcité comme d'un contre-pouvoir pour faire valoir leur droit à préserver la transmission des savoirs en dehors de toute influence religieuse. Leur conduite marque « leur sortie de la religion »: «La "sortie de la religion" ne signifie pas sortie de la croyance religieuse, mais sortie d'un monde où la religion est structurante » (Gauchet, 1998 : 13). En effet, pour la plupart des familles, le cultuel relève de la sphère privée et non de la sphère scolaire (comme c'est le cas dans l'enseignement tunisien avec le cours d'éducation civique et religieuse). C'est la raison pour laquelle des parents veillent même à ce que des imans bénévoles ne viennent plus professer dans les établissements français, même en dehors des heures de cours.

«On m'a dit qu'à un moment donné y avait eu un imam au même titre que le curé, mais finalement il n'a passé qu'un an parce qu'il n'avait pas assez d'élèves, ça n'intéressait pas les familles en fait. J'en ai entendu de nouveau parler parce qu'un professeur d'arabe souhaitait le reproposer et ça a tout de suite été rejeté par l'association de parents d'élèves... » (Monsieur Martin, proviseur).

L'appartenance de classe des familles implique une inscription dans la modernité et des choix culturels dont les valeurs peuvent être portées par la laïcité, une façon, pour elles, de se distinguer et de s'opposer aux goûts des «autres » et de s'opposer à l'islam prosélyte croissant de la sphère publique. C'est pourquoi des parents, pour promouvoir la neutralité des lieux scolaires, vont jusqu'à rejeter hors les murs le moindre "germe » religieux. En effet, aucun compromis religieux n'est concédé malgré le droit de cité de l'aumônerie et le droit, pour les élèves, de recevoir une instruction religieuse s'ils le désirent. Ainsi lorsque l'espace scolaire a abrité bénévolement un enseignant d'instruction islamique, les familles ne lui ont pas envoyé leurs enfants et certaines y ont même vu une atteinte à la liberté religieuse. Des parents ont alors traité avec les membres de l'institution laïque au mieux de leurs intérêts individuels jusqu'à obtenir l'éviction de l'imam.

«Avant y avait un imam, un professeur d'instruction religieuse musulmane qui venait donner des cours deux fois par semaine pour répondre aux questions curieuses des élèves sur la religion. Je sais qu'il n'est même pas resté une année. Au départ il a dit : je viens, je ne demande rien en échange, je viens. Mais il n'y a pas eu une bonne publicité pour motiver les élèves à aller voir l'imam... Et presque aucun élève n'est allé assister à cela, à part un ou deux du premier cycle mais pas les grands... Il est venu plusieurs fois mais il n'y avait pas d'élèves, donc il est parti et personne ne l'a réclamé parce que les parents ils ne veulent pas de lui, hein? »(Madame Shanoun, enseignante d'arabe).

La présence de l'imam a provoqué, chez des parents, un mouvement défensif. Si certains n'étaient probablement pas opposés au bien fondé de sa présence, d'autres, en revanche, l'ont 
refusée en estimant que l'éducation religieuse prodiguée n'était pas conforme à leur vision de l'islam. Monsieur Bendeb, Tunisien de 50 ans, médecin, père d'une lycéenne, explique :

«J'ai voulu inscrire mes filles pour qu'elles aient quelques notions du Coran tout ça, notions je dis bien. Et elles ne sont pas restées longtemps à vrai dire, parce qu'avec ma femme on a décidé de les sortir. Je trouvais inadmissible qu'on fasse un embrigadement, j'estimais que c'était du lavage de cerveau. [...] Mais on n'a pas été les seuls parents à se plaindre... On n'était pas venu chercher un lavage de cerveau hein? Donc finalement, il est parti. Ici nous sommes disons des pratiquants cool et cet imam, il a dépassé les bornes quand il nous a insultés et qu'il a laissé sous-entendre à nos enfants qu'on était de mauvais pratiquants. Ça non! Personne ne viendra me dire quoi faire ou quoi penser. Moi je ne supporte pas cette ardeur des religieux. »

Pour de nombreux parents, en matière de religion, le prosélytisme est à proscrire strictement. En conséquence, comme l'enceinte scolaire laïque sert de contre-pouvoir pour refuser l'islam dogmatique, les établissements français servent la remise en cause de l'autorité religieuse et concourent à la légitimité cultuelle des familles. Pour elles, les valeurs et les pratiques du croyant se vivent plus qu'elles ne s'enseignent. Elles doivent relever d'une attitude éthique, c'est-à-dire d'une capacité de questionnement et d'une acceptation de la diversité, ce qui est finalement incompatible avec une position dogmatique. En conséquence, plutôt que chercher à transmettre à leurs enfants une vision encadrée de l'islam, leur foi est uniquement soumise à leur propre appréciation personnelle, conformément à l'intériorisation des normes familiales.

Nous comprenons donc l'absence d'intérêt qu'a suscité la présence de cet imam dont le bénévolat pouvait être sujet à caution, puisque finalement, la question religieuse, comme fait de culture, semble être l'approche la plus adaptée à la vision du monde de cette population.

Le désintérêt des lycéens et des parents pour un encadrement islamique se fait donc au profit d'une autonomie religieuse qui ne manque pas de susciter critiques et désapprobation de la part de ceux qui sont disposés à suivre le dogme.

«Moi, je pense que c'est intéressant d'apprendre aux élèves la tolérance de l'islam [...]. Je leur dis dans mes cours mais c'est mieux si c'est un vrai professionnel, un professionnel du Coran quand même! [...] Donc il y a des côtés civiques et religieux à apprendre et à donner aux élèves parce que l'enseignement français n'insiste pas sur, heu, la foi. Donc l'imam était nécessaire hein ? Mais bon, tant que la demande ne vient pas des parents on ne peut pas trop insister. Bon, je ne peux pas trop en parler, je n'ai pas le droit de critiquer hein ? Mais quand même ils ne respectent pas bien l'islam, franchement. Ils sont trop trop libres. Ils ont des relations ouvertes, c'est incorrect. Ils se permettent trop de choses. Ils sont en dehors des réalités. [Vous leur en parlez ?] On ne peut rien dire. Il ne faut rien leur dire sinon on a des ennuis avec les parents. » (Monsieur Garaoui, professeur d'arabe).

Pour des enseignants de confession musulmane, l'autorité religieuse doit guider l'expression sociale des lycéens et entretenir leur culture islamique. Or, l'efficacité de ce pouvoir est remise en cause par un autre rapport de domination qui est, nous l'avons énoncé, lié à la position sociale des parents. En effet, ceux-ci veillent à ce que la socialisation religieuse de leurs enfants ne se dérobe pas à l'espace privé de la famille, une façon, pour eux, de recentrer la place fondamentale de la religion. Ainsi, «loin de gêner l'islam, la laïcité a plutôt des effets positifs pour lui. Elle l'oblige à n'être que religion, en lui laissant la possibilité de se développer comme telle» (Barbier, 1995: 211-212). Ces lycéens 
« occidentalisés » se dérobent effectivement, dans leur majorité, aux applications des devoirs islamiques et les parents attendent d'ailleurs des acteurs de l'espace scolaire qu'ils veillent au retranchement prosélyte, faute de quoi, ils interviennent à bras le corps, comme en atteste Madame Sayadi, une architecte tunisienne de 45 ans, musulmane non pratiquante.

«Pas d'islam en intraveineuse dans la famille [Rires] hein ?! Bon, ce n'est pas l'islam de la rue si je puis m'exprimer ainsi. D'ailleurs c'est tout aussi intolérable pour moi lorsque ça se passe ici [dans l'établissement]. [...] Ces dérives [le prosélytisme entre élèves], le lycée doit y remédier, oui c'est son devoir, c'est lä̈que comme système... Bon, bien sûr on est musulman mais on n'est pas rigoriste...Pour qui il se prenait ce môme? C'est nous ses parents. C'est nous l'autorité je dirai. Mon fils connaît sa religion. Ce n'est pas quelque chose qui doit être débattu. »

La visibilité religieuse devient un gage de crédibilité dans la sphère publique et dans certains champs sociaux; à l'inverse, le refus de se plier à cette exigence est assimilé à un affront. Les lycéens renversent donc la donne dans l'espace scolaire laïque en transformant les rapports de domination religieuse. C'est ainsi que des lycéens et des parents, au nom de l'islam et de la modernité, convoquent la laïcité pour contrecarrer les rapports de domination existants. Ils dénoncent la propagande religieuse au nom de la laïcité pour lutter contre le prosélytisme et légitimer leur autonomie cultuelle puisque l'islam est divers et pluriel.

En conclusion, nous pouvons avancer que la laïcité sert les pratiques et les comportements religieux de la population scolaire et des familles. Elles y puisent l'autoprotection ou l'auto préservation de leurs visions de l'islam, islam dont la société civile révèle d'autres pratiques et d'autres valeurs qui engendrent des peurs et des craintes d'embrigadement. Dans ce domaine, le laboratoire que constitue le dispositif scolaire français peut être considéré comme riche d'enseignements puisque cet espace de socialisation témoigne du contournement et du refus du déterminisme religieux de la société tunisienne, en révélant un véritable modèle de coopération entre laïcité et religion, dans le respect même des acquis fondamentaux de la laïcité, bien commun des croyants comme des incroyants.

\section{Bibliographie}

AEFE 2010 L'enseignement français à l'étranger, Tunis, Ministère de l'éducation nationale, direction de l'enseignement scolaire (DESCO), Ministère des affaires étrangères.

BARBIER M. 1995 La laïcité, Paris, L'Harmattan, 312 pages.

BERTAUX D. 2005[1997] L'enquête et ses méthodes. Le récit de vie, Paris, Armand Colin, (2 ${ }^{\mathrm{ème}}$ édition), 128 pages.

BouRdieu P. 1980 Questions de sociologie, Paris, Les Éditions de Minuit, 271 pages.

BouRdieu P. \& WACQUANT L.J.D. 1992 Réponses. Pour une anthropologie réflexive, Paris, Éditions du Seuil, 267 pages.

BORDES-BENAYOUN C. \& TANDIAN A. 2004 Rôles et échanges des savoirs dans la formation des groupes juvéniles urbains, Toulouse, Centre d'Anthropologie - EHESS et Laboratoire Diasporas, 247 pages.

BoucheT G. 1996 Laïcité et enseignement, Paris, Armand Colin, Formation des enseignantsEnseigner, 248 pages.

GAUCHET M.1998 La Religion dans la démocratie. Parcours de la laïcité, Paris, Gallimard, coll. Folio/Essais « Le débat », 130 pages. 
MELLITI I. 2008 "Les jeunes et la religion au Maghreb : entre individualisation et retour à la communauté", in Melliti I., Mahfoudh-Draoui D., Ben Amor R. \& Ben Fredu S. (dir.), Jeunes dynamiques identitaires et frontières culturelles, UNICEF, 68-70.

PAYET J-P. 1999 "Mixité et ségrégations dans l'école urbaine", Hommes et Migrations-1217, $30-42$.

VAn Zanten A. 2009a Choisir son école. Stratégie familiales et médiations locales, Paris, PUF, 283 pages.

VAN ZANTEN A. 2009b "Le choix des autres, Jugements, stratégies et ségrégations scolaires", Actes de la recherche en sciences sociales-180, 24-34. 